\title{
ESTADO NUTRICIONAL ASOCIADO A CARACTERÍSTICAS SOCIODEMOGRÁFICAS EN EL ADULTO MAYOR PERUANO
}

\author{
Carolina Tarqui-Mamani, ${ }^{1,2, a}$, Doris Alvarez-Dongo ${ }^{1, b}$, Paula Espinoza-Oriundo ${ }^{1, b}$, \\ Guillermo Gomez-Guizado $1, \mathrm{c}$
}

\begin{abstract}
RESUMEN
Objetivos. Describir el estado nutricional en adultos mayores y su asociación con características sociodemográficas. Materiales y métodos. Se realizó un estudio transversal analítico. El muestreo fue probabilístico, estratificado y multietápico. El estado nutricional se evalúo mediante el índice de masa corporal (IMC) según la clasificación del estado nutricional para el adulto mayor del Ministerio de Salud de Perú. El análisis estadístico consideró la ponderación necesaria para muestras complejas. Resultados. Se incluyó a 7267 adultos mayores con una media de edad de 70,1 $\pm 8,3$ años. El $26,8 \%$ presentaron delgadez, $21,7 \%$ sobrepeso y $10,6 \%$ obesidad. El analfabetismo (OR 1,9; IC 95\%: 1,2-3,0), nivel educativo primario (OR 1,9; IC 95\%:1,3-2,9), pobreza extrema (OR 2,0; IC 95\%:1,6-2,5), residir en área rural (OR 1,8; IC 95\%:1,5-2,1), sierra (OR 1,6; IC 95\%: 1,2-2,2) o selva (OR 1,6; IC 95\%:1,1-2,2) fueron factores asociados a delgadez. El sexo femenino (OR 1,8; IC 95\%:1,4-2,1), residir en área urbana (OR 2,0; IC 95\%: 1,6-2,5) o costa (OR 1,5; IC 95\%:1,2-1,8); y ser no pobre (OR 1,9; IC 95\%: 1,3-2,9) se asoció a sobrepeso. El sexo femenino (OR 3,1; IC 95\%: 2,3-4,1), nivel educativo primario (OR 2,4; IC 95\%: 1,5-4,0) y secundario (OR 2,0; IC 95\%: 1,2-3,4); vivir en área urbana (OR 2,2; IC 95\%:1,6-2,9), la costa (OR 1,8; IC 95\%: 1,3-2,4), Lima Metropolitana (OR 1,6; IC 95\%:1,1-2,2) selva (OR 1,6; IC 95\%: 1,1-2,2), y ser no pobre (OR 3,5; IC 95\%:1,8-7,0) estuvieron asociados a obesidad. Conclusiones. Los datos sugieren que tanto la delgadez como el exceso de peso son frecuentes en la población de adultos mayores estudiada.
\end{abstract}

Palabras clave: Adulto mayor; Delgadez; Sobrepeso; Obesidad; Epidemiología (fuente: DeCS BIREME).

\section{NUTRITIONAL STATUS ASSOCIATED WITH DEMOGRAPHIC CHARACTERISTICS IN OLDER PERUVIAN ADULTS}

\begin{abstract}
Objectives. To describe the nutritional status in older adults and its association with sociodemographic characteristics. Materials and methods. A cross-sectional study was conducted. Sampling was probabilistic, stratified and multistage. Nutritional status was assessed by body mass index (BMI) according to the classification of nutritional status for the elderly from the Ministry of Health of Peru. The statistical analysis considered the necessary weight for complex samples. Results. The study included 7,267 older adults. $26.8 \%$ of participants were underweight, $21.7 \%$ overweight, $10.6 \%$ obese and $40.8 \%$ normal. The average age in the sample was $70.1 \pm 8.3$ years. Illiteracy (OR 1.9; $95 \% \mathrm{Cl} 1.2-3.0)$, primary education (OR $1.9 ; 95 \% \mathrm{Cl} 1.3-2.9)$, extreme poverty (OR 2.0; $95 \% \mathrm{Cl} 1.6-2.5)$, living in rural areas (OR 1.8; $95 \% \mathrm{Cl} 1.5-2.1)$, living in the mountains (OR 1.6; $95 \% \mathrm{Cl} 1.2-2.2$ ) or jungle (OR 1.6; 95\% Cl 1.1-2.2) were found associated with underweight. Females (OR 1.8; 95\% Cl 1.4-2.1), living in urban areas (OR 2.0; 95\% Cl 1.6-2.5), living in the coastal region (OR 1.5; 95\% Cl 1.21.8); and not classified as poor (OR 1.9; 95\% Cl 1.3-2.9) were associated with overweight. Female sex (OR 3.1; $95 \% \mathrm{Cl}$ 2.3-4.1), primary education (OR 2.4; 95\% Cl 1.5-4.0) and secondary (OR 2.0; 95\% $\mathrm{Cl}$ 1.2-3.4); live in urban areas (OR 2.2; $95 \% \mathrm{Cl} 1.6-2.9)$, inhabiting the coast (OR 1.8; 95\% Cl 1.3-2.4), Metro (OR 1.6, 95\% Cl 1.1-2.2) and jungle (OR 1.6; $95 \% \mathrm{Cl}$ 1.1-2.2), and not classified as poor (OR 3.5; $95 \% \mathrm{Cl}$ 1.8-7.0) were associated with obesity. Conclusions. The data suggest that both underweight and overweight are common in the elderly population studied.
\end{abstract}

Key words: Aged; Thinness; Overweight; Obesity; Epidemiology (source: MeSH NLM).

\footnotetext{
Centro Nacional de Alimentación y Nutrición, Instituto Nacional de Salud. Lima, Perú

Facultad de Medicina, Universidad Nacional Mayor de San Marcos. Lima, Perú.

Obstetra, doctora en salud pública; ${ }^{\mathrm{b}}$ Licenciada en nutrición; ${ }^{\mathrm{c}}$ Médico pediatra.

Recibido: : 17-02-14 Aprobado: 28-05-14
}

Citar como: Tarqui-Mamani C, Alvarez-Dongo D, Espinoza-Oriundo P, Gomez-Guizado G. Estado nutricional asociado a características sociodemográficas en el adulto mayor peruano. Rev Peru Med Exp Salud Publica. 2014;31(3):467-72. 


\section{INTRODUCCIÓN}

El incremento de la población adulta mayor es un fenómeno global. Cerca de 600 millones de personas mayores de 60 años viven en el mundo, y se proyecta que para el 2025 esta cifra se duplicará (1). En América del Sur, la mayor esperanza de vida al nacer se reporta en Chile con 80 años y la menor en Bolivia con 67 años; mientras que en Perú, la esperanza de vida al nacer ha aumentado a 75 años, siendo mayor en mujeres que hombres ${ }^{(2)}$. La población de adultos mayores peruanos actualmente es $9,2 \%$ de la población total y se espera que para el 2021 alcance el $11,2 \%$ de la población total ${ }^{(3)}$.

La creciente urbanización, y la frecuente migración hacia las zonas urbanas contribuyen a incrementar la población adulta mayor, que en el área urbana asciende a $76,7 \%{ }^{(3)}$. El envejecimiento se encuentra en relación directa al desarrollo socioeconómico de la población, debido a las mejoras en las condiciones de vida y acceso a la atención de salud ${ }^{(4)}$. Sin embargo, los adultos mayores son considerados como una población en situación de vulnerabilidad debido a la inseguridad social y económica, así como el alto riesgo de malnutrición que caracteriza a este grupo etario ${ }^{(5-8)}$.

El adulto mayor tiende a consumir menor cantidad de alimentos debido a la disminución de la fuerza de contracción de los músculos de masticación; la reducción progresiva de piezas dentales; la alteración de los umbrales del gusto y el olfato; los cambios en la función motora del estómago; la disminución de los mecanismos de absorción; los estilos de vida inadecuados, como el consumo de alimentos de bajo costo ricos en carbohidratos y pobres en proteínas; por otra parte, el consumo de alcohol y tabaco; la disminución de la actividad física; la posmenopausia; la depresión; el incremento de las enfermedades crónicas, y la mala calidad de vida contribuyen a incrementar la malnutrición en el adulto mayor ${ }^{(9-11)}$.

En América Latina, la prevalencia de desnutrición en ancianos que viven en la comunidad varía entre 4,6 y $18 \%{ }^{(12)}$. La desnutrición aumenta el deterioro de la capacidad funcional, la calidad de vida, y la morbilidad y mortalidad en los ancianos (21,22). Por otro lado, el sobrepeso y la obesidad en este grupo etario, es mayor en el sexo femenino que el masculino, como consecuencia del hipoestrogenismo que caracteriza a la menopausia. Tanto la delgadez como el sobrepeso y la obesidad en los adultos mayores son problemas que se relacionan con la mayor morbilidad y mortalidad de los adultos mayores, ambos, repercuten negativamente sobre la salud del adulto mayor, conllevando a la pérdida de la capacidad funcional y calidad de vida.
El objetivo del estudio fue describir el estado nutricional y determinar las características sociodemográficas asociados en los adultos mayores peruanos.

\section{MATERIALES Y MÉTODOS}

\section{DISEÑO Y POBLACIÓN DE ESTUDIO}

Se realizó un estudio observacional y transversal. Se incluyó a los adultos mayores, residentes en los hogares peruanos donde se llevó a cabo la encuesta y que voluntariamente aceptaron participar en el estudio. Se excluyó a los adultos mayores con limitaciones físicas que dificulten las mediciones antropométricas. El recojo de datos se realizó entre el 2009-2010.

El muestreo fue probabilístico, estratificado, multietápico e independiente en cada departamento del Perú. EI tamaño de la muestra se calculó para estimar algunas características de la vivienda y del hogar, características de los miembros del hogar, educación, salud, empleo, ingreso y gastos del hogar. Se consideró los siguientes criterios: nivel de confianza al 95\%, precisión 5\%, total de viviendas en el Perú, la tasa de no respuesta y el efecto de diseño correspondiente a los reportes previos de la Encuesta Nacional de Hogares (ENAHO). La muestra requerida fue 22640 viviendas. Se seleccionaron aleatoriamente seis viviendas en cada conglomerado urbano y ocho viviendas en el rural ${ }^{(13)}$.

\section{TÉCNICAS E INSTRUMENTOS}

Las mediciones antropométricas se realizaron según metodología del MINSA/INS y los requerimientos técnicos, se utilizó balanzas digitales calibradas con capacidad de $150 \mathrm{k}$ y precisión de $\pm 100 \mathrm{~g}$ y tallímetros móviles de madera. El personal que se encargó de las evaluaciones antropométricas fue capacitado y estandarizado en mediciones de la talla y peso. El estado nutricional se evalúo mediante el índice de masa corporal (IMC) según la clasificación del estado nutricional para el adulto mayor del Ministerio de Salud, delgadez (IMC $\leq 23,0)$, normal $(I M C>23$ a $<28$ ), sobrepeso $(I M C \geq 28 \mathrm{a}<32,0)$ y obesidad $(I M C \geq 32)^{(14)}$.

La pobreza se midió por enfoque monetario (hogar pobre, hogar pobre extremo y no pobre), se consideró: hogar pobre, cuando el gasto per cápita era inferior a la línea de pobreza (hogares que cubren sus necesidades en alimentación, pero no cubren sus necesidades en otros bienes y servicios como educación, salud, vestido, calzado, transporte, vivienda); hogar pobre extremo, cuando el gasto per cápita era inferior a una línea de pobreza extrema (hogares que no pueden cubrir sus 
gastos en alimentos ni sus gastos en otros bienes y servicios como transporte, vestimenta, vivienda, educación y salud), y hogar no pobre, cuando el hogar cubre la canasta familiar que satisface las necesidades nutricionales de las personas y otras necesidades como transporte, vestimenta, vivienda, educación y salud ${ }^{(13)}$.

\section{ANÁLISIS ESTADÍSTICO}

El análisis de los datos se realizó considerando el diseño de muestras complejas y se ajustó por el factor de ponderación. Se calculó las medias, proporciones con intervalo de confianza al 95\%. Se calculó el chi cuadrado y la regresión logística para evaluar la asociación entre las características sociodemográficas con el estado nutricional del adulto mayor. El procesamiento de los datos se realizó mediante el SPSS v22.

\section{CONSIDERACIONES ÉTICAS}

Se solicitó el consentimiento informado a todos los participantes y se acreditó mediante la firma de los participantes o la huella digital en el caso de los analfabetos, este procedimiento se realizó bajo la presencia de un testigo. El estudio se realizó en el marco de la vigilancia de indicadores nutricionales que realiza la Dirección Ejecutiva de Vigilancia Alimentaria y Nutricional del Instituto Nacional de Salud en convenio con el Instituto Nacional de Estadística e Informática y fue registrado y aprobado en el Plan Operativo Institucional del Instituto Nacional de Salud.

\section{RESULTADOS}

Se incluyó 7267 adultos mayores, la media de edad fue $70,1 \pm 8,3$ años con un rango entre 60 a 99 años. El $50,8 \%$ de los participantes fueron mujeres, $20,6 \%$ fueron analfabetas, 45,3\% tuvieron nivel primario, $21,5 \%$ secundario y $12,5 \%$ nivel superior. El $63,6 \%$ tuvieron algún tipo de seguro de salud, 7,4\% fueron pobres extremos y $16,1 \%$ pobres. El $22,9 \%$ vivieron en la zona rural, $37,8 \%$ de los participantes residían en Lima Metropolitana, $23,8 \%$ en la costa, 30,2 en la sierra y $8,2 \%$ en la selva.

El promedio global del IMC fue $26,2 \pm 4,7$ y fue mayor en las mujeres $(26,7)$ que en los varones $(25,6)$ $(p<0,001)$. Del total de adultos mayores, $26,8 \%$ (IC 95\%: 25,4-28,4) tuvieron delgadez; $21,7 \%$ (IC 95\%: 20,3-23,2), sobrepeso; 10,6\% (IC 95\%: 9,5-11,9), obesidad, y el 40,8\% peso normal (IC 95\%: 39,1-42,5). Las características sociodemográficas de los adultos mayores según estado nutricional se presentan en la Tabla 1. A nivel departamental, la prevalencia de delgadez fue mayor en Huánuco $(57,2 \%)$, Ayacucho $(52,8 \%)$, Huancavelica $(52,2 \%)$, Puno (51,6\%) y Cusco $(46,6 \%)$.

Tabla 1. Características de los adultos mayores según estado nutricional. Perú; 2009-2010

\begin{tabular}{|c|c|c|c|c|c|c|c|c|c|c|c|c|c|}
\hline \multirow{2}{*}{ Variables } & \multicolumn{3}{|c|}{ Delgadez } & \multicolumn{3}{|c|}{ Normal } & \multicolumn{3}{|c|}{ Sobrepeso } & \multicolumn{3}{|c|}{ Obesidad } & \multirow{2}{*}{$p$} \\
\hline & $\mathbf{N}$ & $(\%)$ & IC 95\% & $\mathbf{N}$ & $(\%)$ & IC $95 \%$ & $\mathbf{N}$ & $(\%)$ & IC $95 \%$ & $\mathbf{N}$ & $(\%)$ & IC $95 \%$ & \\
\hline \multicolumn{14}{|l|}{ Sexo } \\
\hline Hombre & 1211 & $(27,2)$ & $25,2-29,3$ & 1495 & $(47,1)$ & $44,6-49,6$ & 532 & $(19,4)$ & $17,5-21,5$ & 160 & $(6,3)$ & $5,1-7,8$ & $<0,001$ \\
\hline Mujer & 1309 & $(26,5)$ & $24,7-28,4$ & 1339 & $(35,1)$ & $33,0-37,2$ & 756 & $(23,9)$ & $21,9-26,0$ & 465 & $(14,5)$ & $12,9-16,4$ & \\
\hline \multicolumn{14}{|l|}{ Nivel educativo } \\
\hline Analfabeto & 973 & $(40,7)$ & $37,7-43,7$ & 749 & $(36,7)$ & $33,7-39,7$ & 217 & $(14,3)$ & $11,9-17,0$ & 132 & $(8,4)$ & $6,7-10,5$ & $<0,001$ \\
\hline Primaria & 1275 & $(28,6)$ & $26,5-30,7$ & 1358 & $(38,1)$ & $35,9-40,4$ & 686 & $(21,6)$ & $19,7-23,7$ & 329 & $(11,7)$ & $10,1-13,4$ & \\
\hline Secundaria & 201 & $(16,4)$ & $13,6-19,6$ & 439 & $(43,3)$ & $39,2-47,5$ & 244 & $(27,7)$ & $24,1-31,7$ & 116 & $(12,6)$ & $24,1-31,7$ & \\
\hline Superior & 69 & $(13,1)$ & $9,4-17,8$ & 287 & $(53,9)$ & $47,6-60,1$ & 141 & $(25,6)$ & $20,9-30,9$ & 48 & $(7,4)$ & $5,0-10,8$ & \\
\hline \multicolumn{14}{|l|}{ Área de residencia } \\
\hline Urbano & 788 & $(18,4)$ & $16,7-20,2$ & 1600 & $(42,2)$ & $40,0-44,4$ & 973 & $(26,3)$ & $24,5-28,3$ & 498 & $(13,1)$ & $11,6-14,7$ & $<0,001$ \\
\hline Rural & 1732 & $(51,5)$ & $49,2-53,9$ & 1234 & $(36,6)$ & $34,6-38,6$ & 315 & $(8,3)$ & $7,3-9,5$ & 127 & $(3,6)$ & $2,9-4,4$ & \\
\hline \multicolumn{14}{|c|}{ Dominio geográfico } \\
\hline Lima Metropolitana & 95 & $(14,2)$ & $11,5-17,3$ & 308 & $(43,2)$ & $39,2-47,4$ & 197 & $(28,4)$ & $25,0-32,0$ & 102 & $(14,2)$ & $11,6-17,4$ & $<0,001$ \\
\hline Costa & 354 & $(19,0)$ & $16,5-21,9$ & 732 & $(40,3)$ & $37,3-43,3$ & 496 & $(26,5)$ & $24,0-29,2$ & 277 & $(14,2)$ & $12,1-16,6$ & \\
\hline Sierra & 1628 & $(42,6)$ & $40,3-45,0$ & 1307 & $(38,8)$ & $36,8-40,9$ & 398 & $(13,1)$ & $11,6-14,7$ & 167 & $(5,5)$ & $4,6-6,5$ & \\
\hline Selva & 443 & $(36,7)$ & $32,9-40,6$ & 487 & $(40,4)$ & $37,3-43,5$ & 197 & $(16,1)$ & $13,6-19,0$ & 79 & $(6,9)$ & $5,0-9,3$ & \\
\hline \multicolumn{14}{|l|}{ Pobreza } \\
\hline Pobreza extrema & 581 & $(61,5)$ & $57,1-65,7$ & 278 & $(31,9)$ & $28,1-36,0$ & 45 & $(5,3)$ & $3,7-7,4$ & 11 & $(1,3)$ & $0,7-2,5$ & $<0,001$ \\
\hline Pobreza & 659 & $(40,0)$ & $36,4-43,7$ & 503 & $(38,9)$ & $35,3-42,5$ & 159 & $(13,8)$ & $11,3-16,7$ & 73 & $(7,4)$ & $5,4-9,9$ & \\
\hline No pobre & 1280 & $(20,4)$ & $18,9-22,0$ & 2053 & $(42,1)$ & $40,1-44,2$ & 541 & $(25,1)$ & $23,4-27,0$ & 541 & $(12,3)$ & $10,9-13,8$ & \\
\hline
\end{tabular}


Tabla 2. Análisis multivariado de las características sociodemográficas y el estado nutricional del adulto mayor. Perú; 2009-2010

\begin{tabular}{|c|c|c|c|c|c|c|}
\hline \multirow{2}{*}{ Variables } & \multicolumn{2}{|c|}{ Bajo peso* } & \multicolumn{2}{|c|}{ Sobrepeso* } & \multicolumn{2}{|c|}{ Obesidad* } \\
\hline & OR & (IC 95\%) & OR & (IC 95\%) & OR & IC 95\%) \\
\hline \multicolumn{7}{|l|}{ Sexo } \\
\hline Hombre & 1,0 & - & 1,0 & - & 1,0 & - \\
\hline Mujer & 1,2 & $(1,0-1,4)$ & 1,8 & $(1,4-2,1)$ & 3,1 & $(2,3-4,1)$ \\
\hline \multicolumn{7}{|l|}{ Nivel educativo } \\
\hline Analfabeto & 1,9 & $(1,2-3,0)$ & 1,0 & $(0,7-1,5)$ & 1,8 & $(1,0-3,1)$ \\
\hline Primaria & 1,9 & $(1,3-2,9)$ & 1,4 & $(1,0-1,9)$ & 2,4 & $(1,5-4,0)$ \\
\hline Secundaria & 1,4 & $(0,9-2,3)$ & 1,3 & $(0,9-1,9)$ & 2,0 & $(1,2-3,4)$ \\
\hline Superior & 1,0 & - & 1,0 & - & 1,0 & - \\
\hline \multicolumn{7}{|l|}{ Área** } \\
\hline Urbano & 1,0 & - & 2,0 & $(1,6-2,5)$ & 2,2 & $(1,6-2,9)$ \\
\hline Rural & 1,8 & $(1,5-2,1)$ & 1,0 & - & 1,0 & - \\
\hline \multicolumn{7}{|l|}{ Región natural** } \\
\hline $\begin{array}{l}\text { Lima } \\
\text { Metropolitana }\end{array}$ & 1,0 & - & 1,3 & $(1,0-1,7)$ & 1,6 & $(1,1-2,2)$ \\
\hline Costa & 1,1 & $(0,8-1,5)$ & 1,5 & $(1,2-1,8)$ & 1,8 & $(1,3-2,4)$ \\
\hline Sierra & 1,6 & $(1,2-2,2)$ & 1,0 & - & 1,0 & - \\
\hline Selva & 1,6 & $1,1-2,2)$ & 1,1 & $(0,8-1,4)$ & 1,6 & $(1,1-2,2)$ \\
\hline \multicolumn{7}{|l|}{ Pobreza ${ }^{* *}$} \\
\hline Pobre extremo & 2,0 & $(1,6-2,5)$ & 1,0 & - & 1,0 & - \\
\hline Pobre & 1,4 & $(1,2-1,7)$ & 1,3 & $(0,9-2,1)$ & 2,5 & $(1,2-5,2)$ \\
\hline No pobre & 1,0 & - & 1,9 & $(1,3-2,9)$ & 3,5 & $(1,8-7,0)$ \\
\hline
\end{tabular}

*Se consideró el estado nutricional normal para la referencia nutricional ** La regresión logística se realizó en forma independiente (bajo peso, sobrepeso u obesidad), por ello, las categorías de referencia difieren según el estado nutricional.

En los resultados de la regresión logística se encontró como factores sociodemográficos asociados con la delgadez en el adulto mayor al nivel educativo primario (OR 1,9; IC 95\%:1,3-2,9), la pobreza extrema (OR 2,0; IC $95 \%: 1,6-2,5)$, residir en el área rural (OR 1,8; IC $95 \%: 1,5-2,1)$, la sierra (OR 1,6; IC 95\%: 1,2-2,2) o la selva (OR 1,6; IC 95\%:1,1-2,2). Como factores asociados al sobrepeso el sexo femenino (OR 1,8; IC 95\%:1,4-2,1), la condición de no pobre (OR 1,9; IC 95\%: 1,3-2,9), residir en el área urbana (OR 2,0; IC 95\%: 1,6-2,5), costa (OR $1,5$; IC $95 \%: 1,2-1,8)$ y como factores sociodemográficos asociados con la obesidad el sexo femenino (OR 3,1; IC 95\%: 2,3-4,1), el nivel educativo primario (OR 2,4; IC 95\%: 1,5-4,0) o secundario (OR 2,0; IC 95\%: 1,2-3,4), la condición de no pobre (OR 3,5; IC 95\%:1,8-7,0), residir en el área urbana (OR 2,2; IC 95\%:1,6-2,9), costa (OR 1,8; IC 95\%: 1,3-2,4), Lima Metropolitana (OR 1,6; IC 95\%: 1,1-2,2) o selva (OR 1,6; IC 95\%: 1,1-2,2) (Tabla 2).

\section{DISCUSIÓN}

Los resultados muestran que la cuarta parte de los adultos mayores tuvieron delgadez y la tercera parte tuvo sobrepeso u obesidad, y constituirian un problema alto de salud pública en la población de adultos mayores peruanos.

La prevalencia de delgadez en los adultos mayores peruanos es tres veces mayor que la reportada en otras poblaciones de adultos mayores, por ejemplo, los adultos mayores chilenos $(8,4 \%)$, libaneses $(7,6 \%)$, pero dos veces mayor que los adultos mayores brasileños $(10 \%)$ y un poco menor que los adultos mayores cubanos (33\%) (15-18), la delgadez es similar entre los hombres y las mujeres adultos mayores peruanos, a diferencia de un estudio en Brasil, en quienes se observa que la delgadez es mayor en varones ${ }^{(19)}$. Una posible explicación de la elevada prevalencia de delgadez en los adultos mayores peruanos es la situación de abandono familiar, el deterioro de las piezas dentarias y el aumento de las enfermedades crónicas como la artrosis, la hipertensión arterial y la diabetes ${ }^{(12,20)}$.

La quinta parte de los adultos mayores tuvieron sobrepeso, resultado que difiere de los adultos mayores chilenos $(28,9 \%)$. La prevalencia de obesidad de los adultos mayores fue mayor que la obesidad en los adultos mayores chilenos $(7,1 \%)$ y cubanos, pero tres veces menor que la obesidad en los libaneses, sin embargo, fue menor que los adultos mayores cubanos $(29,6 \%)$ y en la población adulta mayor peruana ${ }^{(15,16)}$. Entre las posibles explicaciones podemos mencionar: el abandono familiar y la menor percepción de ingresos económicos, lo cual favorece el consumo de alimentos de bajo costo que son ricos en carbohidratos; por otro lado, la disminución de la actividad física, las enfermedades crónicas, la depresión, la apatía, y el dejar de fumar contribuyen a incrementar el sobrepeso y la obesidad ${ }^{(20,21)}$.

El sobrepeso y la obesidad fueron mayores en las mujeres que en los hombres, resultados similares se observó en los adultos mayores brasileños y peruanos $(18,19,22-24)$. Entre las posibles explicaciones, se puede mencionar que la menopausia favorece el acúmulo graso, que se atribuye al hipoestrogenismo, debido a que los estrógenos estimulan la producción de la leptina que es una proteína segregada en el tejido adiposo que informa al cerebro las reservas energéticas, se sabe que en las mujeres en edad fértil los niveles de leptina son más elevados durante la fase lútea y su concentración declina tras la menopausia ${ }^{(25,26)}$. Los estrógenos también regulan el apetito ${ }^{(27)}$, al parecer la sensación de saciedad estimulada por colecistoquinina aumenta por los estrógenos, por otro lado, los niveles de galanina que son estimulantes de la ingesta de grasas, se encuentra incrementado y los neuropéptidos " $Y$ " están disminuidos en las menopaúsicas comparado a las mujeres en edad fértil. Algunos autores refieren que 
el primer embarazo muy temprano, los periodos cortos de lactancia o ganancia de peso excesivo durante las gestaciones contribuyen a incrementar el sobrepeso y la obesidad durante la menopausia $(27,28)$.

Se observó una relación inversamente proporcional entre el nivel educativo y delgadez y, contrariamente, una relación directa con el sobrepeso y obesidad. Los resultados tienen similitud con lo reportado por Boscatto, quien reportó que la delgadez fue más prevalente en los adultos mayores brasileños analfabetos y el sobrepeso en los alfabetos ${ }^{(19)}$. Entre las posibles explicaciones se puede mencionar que el analfabetismo está vinculado a la pobreza, y los adultos mayores analfabetos y pobres podrían encontrarse en situación de abandono, menores ingresos que limitarían el acceso a alimentos que cubran los requerimientos nutricionales, por otra parte, los adultos mayores analfabetos y pobres tienen tendencia a escoger alimentos más baratos, que pueden ser ricos en carbohidratos pero pobres en proteínas ${ }^{(28)}$. Los resultados muestran que a medida que aumenta el nivel educativo aumenta el sobrepeso y la obesidad, aunque esta última muestra una tendencia creciente hasta el nivel secundario, posteriormente disminuye en el nivel educativo superior. Una posible explicación al incremento del sobrepeso podría ser que los adultos mayores con mayor nivel educativo perciben ingresos por la jubilación, el mejor nivel socioeconómico incrementa el acceso y consumo de alimentos con alto contenido energético, mayor desplazamiento a través del transporte privado y menor actividad física, por tanto, mayor tendencia a un balance positivo entre la ingesta de calorías y el gasto energético.

La delgadez fue más prevalente en los adultos mayores que residen en el área rural y con pobreza lo que podría atribuirse a la actividad laboral que desarrollan como las actividades agrícolas y ganaderas, las cuales demandan mayor gasto energético, a diferencia de las zonas urbanas, en donde predomina las actividades laborales que demandan menor gasto energético; por otro lado, la disponibilidad de los alimentos, su acceso y su consumo determinan la alimentación de la población. Paralelamente, se observa que el sobrepeso y la obesidad es más frecuente en Lima Metropolitana y la costa, regiones que se caracterizan por el mayor desarrollo económico, aumento masivo de los medios de transporte y la proliferación de los alimentos industrializados, las "comidas rápidas" y la baja actividad física, lo cual contribuye al incremento del sobrepeso y la obesidad.

La asociación encontrada entre la delgadez, sobrepeso, obesidad y las principales características sociodemográficas de esta población, son consistentes con otros estudios ${ }^{(16,19,29)}$ siendo una causa importante los hábitos de alimentación de la población peruana que tiene como preferencia el consumo de alimentos con alto valor calórico provenientes principalmente de carbohidratos y en menor grado, por los alimentos como verduras y frutas. Por ejemplo, en la sierra, predomina el consumo de papa, chuño, habas, trigo y fideos; mientras que en la selva existe mayor preferencia por el consumo de yuca, arroz, fideo, frijoles ${ }^{(30)}$.

Una limitación del estudio fue el tamaño de la muestra, el cual fue calculado por el Instituto Nacional de Estadística e Informática para evaluar la pobreza, las condiciones de vida y algunas características de salud en los hogares. Sin embargo, el total de adultos mayores evaluados fue considerable a nivel nacional y departamental, por lo cual, permitirían mostrar una aproximación del estado nutricional de este grupo etario. El uso del índice de masa corporal en los adultos mayores podría subestimar la delgadez y sobreestimar el sobrepeso debido a la posible reducción de la talla, el encogimiento vertical y la curvatura de la columna vertebral del adulto mayor, no obstante, se empleó equipos calibrados y el personal que se encargó de las evaluaciones antropométricas fueron profesionales, estandarizados en precisión y técnica a fin de garantizar la calidad de la información.

En conclusión, la cuarta parte de los adultos mayores tuvieron delgadez y la tercera parte sobrepeso u obesidad. Las principales características sociodemográficas de los adultos mayores peruanos estuvieron asociadas con delgadez, el sobrepeso y la obesidad. Los resultados ponen de manifiesto la necesidad de implementar programas de salud que incluya la evaluación nutricional periódica e individualizada, con el objetivo de mejorar la calidad de vida de esta población.

Agradecimientos: al equipo técnico de supervisión, bioquímica y antropometría de la Dirección Ejecutiva de Vigilancia Alimentario y Nutricional del Centro Nacional de Alimentación y Nutrición del INS.

Contribuciones de autoría: CBTM ha participado en la concepción del artículo, procesamiento de datos, análisis, redacción y aprobación de la versión final. DAD participó en la redacción y aprobó la versión final. PLEO aprobó la versión final del artículo y GGG participó en el análisis, redacción y aprobó la versión final.

Fuentes de financiamiento: este estudio fue financiado por el Instituto Nacional de Salud y se realizó en el marco del convenio suscrito entre el Instituto Nacional de Estadística e Informática y el Instituto Nacional de Salud.

Conflictos de interés: los autores declaran no tener conflictos de interés. 


\section{REFERENCIAS BIBLIOGRÁFICAS}

1. United Nations. Department of Economic and Social Affairs, Population Division. World population ageing 1950-2050. New York: UN; 2002.

2. Organización Panamericana de la Salud; Organización Mundial de la Salud. Enfermedades transmisibles y análisis de salud/información y análisis de Salud: Situación de salud en las Américas: Indicadores Básicos 2013. Washington, D.C: OPS; 2013.

3. Instituto Nacional de Estadística e Informática. Día mundial de la población. Lima: INEI; 2013.

4. Organización Mundial de la Salud. La buena salud añade vida a los años: información general para el Día Mundial de la Salud 2012. Ginebra: OMS; 2012.

5. Cervantes L, Montoya M, Núñez L, Borges A, Gutiérrez-Robledo LM. Aporte dietético de energía y nutrimentos en adultos mayores de México. Nutr Clin. 2003;6:2-8.

6. Arzate J, Fuentes G, Retel C. Desigualdad y vulnerabilidad en el colectivo de Adultos mayores en México y el Estado De México: Una revisión multidisciplinaria. Quivera. 2007;9(2):231-62.

7. Brownie $S$. Why are elderly individuals at risk of nutritional deficiency? Int J Nurs Pract. 2006 Apr;12(2):110-8.

8. Lorefält B, Andersson A, Wirehn AB, Wilhelmsson S. Nutritional status and health care costs for the elderly living in municipal residential homes - an intervention study. J Nut Health Aging. $2011 \mathrm{Feb} ; 15(2): 92-7$.

9. Zayas E. Consideraciones sobre la nutrición en la tercera edad. Nut Clin. 2004;7(2):131-4.

10. Inelmen E, Sergi G, Coin A, Miotto F, Peruzza S, Enzi G. Can obesity be a risk factor in elderly people?. Obes Rev. 2003 Aug;4(3):147-55.

11. Gutiérrez J, Serralde A, Guevara M. Prevalencia de desnutrición del adulto mayor al ingreso hospitalario. Nutr Hosp. 2007;22(6):702-9.

12. Franco-Álvarez N, Ávila-Funes J, Ruiz-Arreguí L, Gutiérrez-Robledo L. Determinantes del riesgo de desnutrición en los adultos mayores de la comunidad: análisis secundario del estudio Salud, Bienestar y Envejecimiento (SABE) en México. Rev Panam Salud Publica. 2007;22(6):36975.

13. Instituto Nacional de Estadística e Informática (INEI). Perú, Encuesta Nacional de Hogares sobre Condiciones de Vida y Pobreza 2011, condiciones de vida y pobreza. Lima: INEI; 2011.

14. Instituto Nacional de Salud. Guía técnica para la valoración nutricional antropométrica de la persona adulta mayor. Lima: INS; 2013.

15. Barrón V, Mardones A, Vera S, Ojeda G, Rodríguez F. Comparación de estilos de vida de la población económicamente activa mayor de 65 años entre las ciudades de Chillán y Valparaíso, Chile. Theoria. 2006;15(1):33-44.

16. Boulos C, Salameh P, Barberger-Gateau P. The AMEL study, a cross sectional population-based survey on aging and malnutrition in 1200 elderly Lebanese living in rural settings: protocol and sample characteristics. BMC Public Health. 2013 Jun 12;13:573. doi: 10.1186/1471-2458-13-573.

17. Pereira R Santa Cruz M. Risk of malnutrition among brazilian institutionalized elderly: a study with the mini nutritional assessment (MNA) questionnaire. J Nutr Health Aging. 2011 Aug;15(7):532-5.

18. Da Silva Coqueiro, Rodrigues Barbosa A, Ferreti Borgatto A. Nutritional status, health conditions, and sociodemographic factors in the elderly of Havana, Cuba: data from SABE survey. J Nutr Health Aging. 2010 Dec;14(10):803-8.

19. Boscatto EC, Duarte Mde F, Coqueiro Rda S, Barbosa AR. Nutritional status in the oldest elderly and associated factors. Rev Assoc Med Bras. 2013 JanFeb;59(1):40-7.

20. Instituto Nacional de Estadística e Informática (INEI). Situación de la niñez y del adulto mayor Julio-AgostoSetiembre 2013. Lima: INEI; 2013.

21. Heikkinen RL, Berg S, Avlund K, Tömäkangas T. Depressed mood: changes during a five-year follow-up in 75-year-old men and women in three Nordic localities. Aging Clin Exp Res. 2002 Jun;14(3 Suppl):16-28.
22. Silveira EA, Kac G, Barbosa LS [Obesity prevalence and associated factors in the elderly in Pelotas, Rio Grande do Sul state, Brazil: obesity classification according to two cutoff points for body mass index]. Cad Saude Publica. 2009 Jul;25(7):1569-77. [Article in Portuguese].

23. Barbosa AR, Souza JM, Lebrão ML, de Fátima Nunes Marucci M. [Nutritional status and physical performance of elderly in the city of São Paulo]. Rev Assoc Med Bras. 2007 Jan-Feb;53(1):75-9. [Article in Portuguese].

24. Instituto Nacional de Salud. Encuesta Nacional de Indicadores Nutricionales, Bioquímicos, Socioeconómicos y Culturales Relacionados con las Enfermedades Crónicas Degenerativas. Lima: INS; 2006.

25. Tommaselli GA, Di Carlo C, Pellicano M, Nasti A, Ferrara C, Di Spiezio Sardo A et al. Modificazioni dei livelli sierici di leptina in menopausa. Minerva Ginecol. 2001 Jun;53(3):193-8.

26. Pavón de Paz I, Alameda C, Olivar J. Obesidad y menopausia. Nutr Hosp. 2006;21(6):633-7.

27. Milewicz A, Tworowska U, Demissie M. Menopausal obesity-myth or fact? Climateric 2001 Dec;4(4):273-83.

28. LovejoyJC. The menopause and obesity. Prim Care. 2003 Jun;30(2):317-25.

29. Instituto Nacional de Salud. Encuesta Nacional de Indicadores Nutricionales, Bioquímicos, Socioeconómicos y Culturales Relacionados con las Enfermedades Crónicas Degenerativas. Lima: INS; 2006.

30. Instituto Nacional de Salud. Reporte de la Encuesta Nacional de Consumo familiar de alimentos. Lima: INS; 2006.

Correspondencia: Carolina Tarqui Mamani Dirección: Jr. Tizón y Bueno 276, Jesús María, Lima 11, Lima, Perú.

Correo electrónico: carobtm@gmail.com, ctarqui@ins.gob.pe 